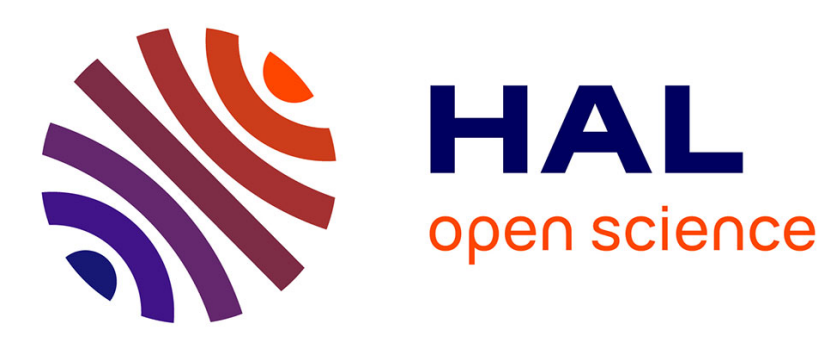

\title{
New Class of High Frequency Nonlinear Magnetic Oscillations in Epitaxial Garnet Films
}

\author{
D. Romanov, E. Rudashevsky, E. Nikolaev
}

\section{To cite this version:}

D. Romanov, E. Rudashevsky, E. Nikolaev. New Class of High Frequency Nonlinear Magnetic Oscillations in Epitaxial Garnet Films. Journal de Physique IV Proceedings, 1997, 07 (C1), pp.C1-463-C1464. 10.1051/jp4:19971189 . jpa-00254838

\section{HAL Id: jpa-00254838 https://hal.science/jpa-00254838}

Submitted on 1 Jan 1997

HAL is a multi-disciplinary open access archive for the deposit and dissemination of scientific research documents, whether they are published or not. The documents may come from teaching and research institutions in France or abroad, or from public or private research centers.
L'archive ouverte pluridisciplinaire HAL, est destinée au dépôt et à la diffusion de documents scientifiques de niveau recherche, publiés ou non, émanant des établissements d'enseignement et de recherche français ou étrangers, des laboratoires publics ou privés. 


\title{
New Class of High Frequency Nonlinear Magnetic Oscillations in Epitaxial Garnet Films
}

\author{
D.A. Romanov, E.G. Rudashevsky and E.I. Nikolaev* \\ General Physics Institute, Vavilov st. 38, Moscow 117942, Russia \\ * Physical and Technical Institute, Roza Luxemburg st.72, Donetsk 340114, Ukraine
}

\begin{abstract}
We performed magneto-optical investigations of the shape of the magnetization precession trajectory in garnet films at large precession angle. To describe the precession in asymmetric potential well the second order terms of small magnetization deflections are taken into account in the expansion of the thermodynamical potential. It is shown, that zero and second harmonic appear in the magnetization oscillations spectrum. The amplitude of this harmonics are proportional to the square of the exciting field amplitude. The dependence of asymmetric precession degree upon applied field and film parameters is found. The reported effects are like second harmonic generation and light detection effects in nonlinear optics.
\end{abstract}

\section{INTRODUCTION}

As known, the nonlinear effects in high frequency magnetization dynamics are connected with interaction between a various types of magnetization oscillations and/or with external exciting field. As a rule, the nonlinear properties of magnetic media are not taken into consideration, since the amplitude of magnetic oscillation is small. It is assumed in this approximation, that the magnetization is located in symmetric parabolic potential well and that the trajectory of precession is ellipse. The maximum angle of precession, achieved in FMR experiments, can be estimated as $\theta_{\max } \approx 0.5 \sqrt{\Delta H / 4 \pi M_{s}}$, where $\Delta H$ is a FMR line width and $4 \pi M_{s}$ is a saturation magnetization [1]. In pure YIG films $\Delta H=0.5 \mathrm{Oe}, 4 \pi M_{3}=1750 \mathrm{Oe}$, consequently, $\theta_{\max } \sim 10^{-2} \mathrm{rad}$. The opposite is true in bubble type garnet films: $\Delta H$ and $4 \pi M_{s}$ may be of the same order of magnitude and $\theta_{\max } \sim 1 \mathrm{rad}$. In this case the cubic anisotropy of garnet film may affect very strongly the magnetization precession: the shape of precession trajectory may be asymmetric and depend upon exciting magnetic field [2]. The results of theoretical and experimental investigations of the magnetization dynamics in asymmetric potential well at large precession angle are presented here.

\section{THEORY}

The variable part of thermodynamical potential for bubble type garnet film is

$F=-M H\left(\sin \theta \sin \theta_{H} \cos \left(\varphi-\varphi_{H}\right)+\cos \theta \cos \theta_{H}\right)+\left(2 \pi M^{2}-K_{0}\right) \cos ^{2} \theta+K_{1}\left(\frac{\sin ^{4} \theta}{4}+\frac{\cos ^{4} \theta}{3}+\frac{\sqrt{2}}{3} \sin ^{3} \theta \cos \theta \cos 3 \varphi\right)-M h \cos \theta$

where $K_{0}, K_{1}$ are constants of uniaxial and cubic anisotropy, $\theta, \theta_{H}, \varphi, \varphi_{H}$ are polar and azimuthal angles of magnetization $\boldsymbol{M}$ and magnetic applied field $\boldsymbol{H}$, respectively. The film normal is parallel to a [111] axis. The polar and azimuthal angles are counted from [111] and [11 $\overline{2}]$ directions, respectively. The of magnetic field $h$ is normal to the film plane. The Landau-Lifshit $z$ equation in polar coordinates has a form

$$
\dot{\theta}=-\frac{\gamma}{M \sin \theta} F_{\varphi}-\frac{\omega_{d}}{M^{2}} F_{\theta} ; \dot{\varphi}=-\frac{\omega_{d}}{M^{2} \sin ^{2} \theta} F_{\varphi}+\frac{\gamma}{M \sin \theta} F_{\theta}
$$

where $\gamma$ is gyromagnetic ratio, $\omega_{d}$ is a dissipation parameter, $F_{\theta}, F_{\varphi}$ denote the first derivative of $F$ by $\theta$ or $\varphi$. Including in the consideration the second order terms of small deviations of the magnetization $\Delta \theta, \Delta \varphi$ from an equilibrium orientation of magnetization $\theta_{0}, \varphi_{0}$ in the expansion of $F_{\theta}, F_{\varphi}$ we obtain the system of nonlinear differential equations with some constant coefficients:

$$
\dot{x}=A_{1} x+B_{1} y+C_{1} x^{2}+D_{1} x y+E_{1} y^{2}+h_{1} \sin \omega t ; \dot{y}=A_{2} x+B_{2} y+C_{2} x^{2}+D_{2} x y+E_{2} y^{2}+h_{2} \sin \omega t ; \quad x=\Delta \theta, y=\Delta \varphi
$$

Solution of this system was performed by means of perturbation technique. Then $\Delta \theta, \Delta \varphi$ takes the next form:

$$
\Delta \theta=a_{0}+a_{1} \sin \omega t+a_{2} \cos \omega t+a_{3} \sin 2 \omega t+a_{4} \cos 2 \omega t+\ldots ; \quad \Delta \varphi=b_{0}+b_{1} \sin \omega t+b_{2} \cos \omega t+b_{3} \sin 2 \omega t+b_{4} \cos 2 \omega t+\ldots
$$


The coefficients $a_{1}, a_{2}, b_{1}, b_{2}$ are proportional to $h$ and depend upon the second derivatives of $F$. They define the elliptic trajectory of magnetization (the "standard" FMR). The coefficients $a_{0}, b_{0}$ are proportional to $h^{2}$ and depend upon the third derivatives of $F$. They define a shift of trajectory from equilibrium orientation of $\boldsymbol{M}$. The coefficients $a_{3}, a_{4}, b_{3}, b_{4}$ are also proportional to $h^{2}$ and depend upon the third derivatives of $F$. They define a second harmonic generation effect. These nonlinear effects in magnetic oscillations are due to nonlinear properties of the magnetic media and have unthreshold nature. Let us consider two cases:

a) $M$ is directed along $[1 \overline{1} 0]$ axis (symmetric potential well): $\theta_{0}=\varphi_{0}=\theta_{H}=\varphi_{H}=\pi / 2$. In this case all third derivatives of $F$ are equal to zero and the magnetization precession trajectory is an ellipse.

b) $M$ is directed along [ 1$] \overline{2}$ ] axis (asymmetric potential well): $\theta_{0}=\pi / 2, \varphi_{0}=0, \cos \theta_{H}=\sqrt{2} K_{1} / 3 M H, \varphi_{H}=0$.

The precession trajectory are shown in the Figure 1 for a number of exciting field amplitudes. In this case a independent from oscillation amplitude parametr $q=a_{6} /\left(a_{1}^{2}+a_{2}^{2}\right)$ is conveniently defined to describe the asymmetric precession degree. Then $q^{-1}$ has a sence the symmetric precession degree:

$$
q^{-1} \approx-\frac{4 \sqrt{2}}{3 H_{k}}\left(H+4 \pi M_{s}-H_{a}-\frac{H_{k}}{2}\right)\left(1+\frac{\omega^{2}}{\gamma^{2} H^{2}}\right)^{-1} ; \quad H_{a}=\frac{2 K_{0}}{M} ; \quad H_{k}=\frac{2 K_{1}}{M}
$$

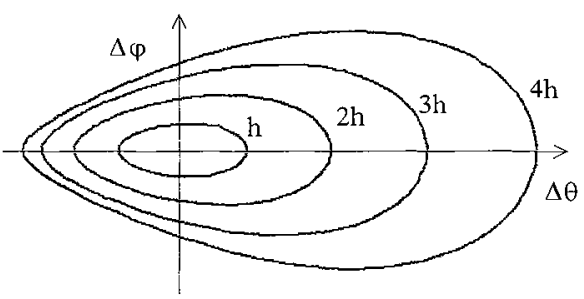

Figure 1: Precession in asymmetric potential well.

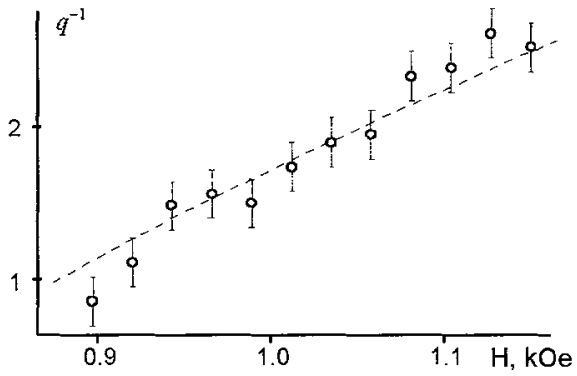

Figure 2: Symmetric degree vs applied field in uniform state.

\section{EXPERIMENT}

To measure of this new nonlinear effects we used the FMR magnetooptical detection technique [3]. The basic idea of the magnetooptical detection is: the asymmetric precession of magnetization leads to asymmetric changes in the polarization of the light, transmitted through the sample. The dependence asymmetric degree upon $H$ was measured in uniaxial garnet film in the vicinity of phase transition from uniform state to domain state. The film had the following parameters: $4 \pi M_{s}=40 \mathrm{G}, H_{a}=890 \mathrm{Oe}, H_{k}=-230 \mathrm{Oe}, \gamma=1.08 \cdot 10^{7}(s \cdot O e)^{-1}, \Delta H=30 \mathrm{Oe}$; film thickness $d=6 \mu \mathrm{m}$.

\section{RESULTS AND DISCUSSION}

The experimental and theoretical results are in good agreement (Figure 2). The angle $\theta_{\max }$ in real experiments was $\sim 0,1$ rad. So, as shown in this paper, the precession of magnetization in the asymmetric potential well leads to a new nonlinear effects. This effects is due to the symmetry of magnetic crystal and has unthreshold nature. To our opinion, they were not observed earlier by next reasons. First, the standard if FMR spectrometer can not detect the asymmetric precession. Second, the nonlinear properties of the magnetic media are not visible at background of the "standard" nonlinear effects. At last, the FMR experiments in garnet films are performed (as a rule) at frequency $\sim 9 \mathrm{GHz}$ where $\mathrm{H}>>\mathrm{H}_{k}$. It should be noted, that reported effects are like second harmonic generation and light detection effects in nonlinear optics.

\section{Acknowledgements}

The authors are grateful to N.M.Abramova and K.S.Juranova for preparation of the sample. Authors are very thankful to Russian Fond of Basic Research (grant 96-02-17706).

\section{References}

[1] Lax B., Button K.J., Microwave ferrites and ferrimagnetics (McGraw-Hill Book Company, Inc., New-York, 1962).

[2] Romanov D.A., Rudashevsky E.G., Mukhamediarov V.A. and Nikolaev E.I., "The discovery of the asymmetric magnetization precession in crystals.", the abstracts of ICM'94 Warsaw 2I-26 August 1994, p.250.

[3] Romanov D.A., Rudashevsky E.G. and Nikolaev E.I., IEEE Trans. Magn. 29 (1993) 3405-3407. 